\title{
Detection of spin coherence in cold atoms via Faraday rotation fluctuations
}

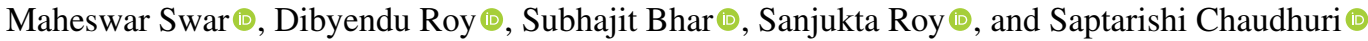 \\ Raman Research Institute, C. V. Raman Avenue, Sadashivanagar, Bangalore 560080, India
}

(Received 14 May 2021; accepted 11 November 2021; published 10 December 2021)

\begin{abstract}
We report noninvasive detection of spin coherence in a collection of Raman-driven cold atoms using dispersive Faraday rotation fluctuation measurements, which opens possibilities of probing spin correlations in quantum gases and other similar systems. We demonstrate five orders of magnitude enhancement of the measured signal strength as compared to traditional spin noise spectroscopy with thermal atoms in equilibrium. Our observations are in good agreement with the comprehensive theoretical modeling of the driven atoms at various temperatures. The extracted spin relaxation rate of cold rubidium atoms with atom number density $\sim 10^{9} / \mathrm{cm}^{3}$ is of the order of $3 \times 10^{3} \mathrm{~s}^{-1}$ at $150 \mu \mathrm{K}$, two orders of magnitude less than $3 \times 10^{5} \mathrm{~s}^{-1}$ of that of a thermal atomic vapor with atom number density $\sim 10^{12} / \mathrm{cm}^{3}$ at $373 \mathrm{~K}$.
\end{abstract}

DOI: 10.1103/PhysRevResearch.3.043171

\section{INTRODUCTION}

The prospects of noninvasive measurement schemes have found increasing research interest in recent decades to detect equilibrium and nonequilibrium properties of microscopic and mesoscopic quantum system [1,2]. Modern scientific disciplines, in particular, quantum information science [3], quantum sensing [4], and metrology [5] can take advantage of the direct applications of these nondestructive measurements. Optical measurements employing dispersive light-matter interactions such as detection of the Faraday or Kerr rotation using an off-resonant probe light are examples of such measurements that disturb the measured samples minimally. Nondestructive optical Faraday and Kerr rotation measurements have been proposed and applied to a broad range of systems, including the readout of a single electron's spin state in a semiconductor quantum dot [6-8] and the quantum gas microscope [9-13] for site-resolved imaging of single isolated atoms in an optical lattice.

In the absence of finite magnetization along the light propagation direction, dynamical magnetic properties of the sample can be found from the temporal fluctuations of dispersive Faraday rotation. Such Faraday rotation noises have been extensively studied within the spin noise spectroscopy (SNS) [14-16] technique to detect the intrinsic spin dynamics in atomic vapors [17-20], semiconductor heterostructures [21], quantum dots [22,23], spin-exchange collisions [24,25], and exciton-polaritons [26,27]. SNS is also applied for precision magnetometry by using a spectral resolution of the spin noise (SN) signals from thermal atomic vapors $[17,18]$ or semiconductors [28].

Published by the American Physical Society under the terms of the Creative Commons Attribution 4.0 International license. Further distribution of this work must maintain attribution to the author(s) and the published article's title, journal citation, and DOI.
However, Faraday rotation fluctuation signals have not been detected in ultracold atoms and quantum gases, where direct measurement of spin fluctuations is predicted to be extremely useful in understanding quantum phases [29-32] and precision magnetometry [33,34]. Moreover, cold atomic systems are ideal test beds for demonstrating quantum effects due to their ultralow temperatures [35].

In this paper, we focus on detecting spin coherence in cold atoms using Faraday-rotation fluctuation measurements. We theoretically develop and experimentally realize this measurement method to demonstrate an enhancement of the signal strength as much as $10^{5}$ in thermal atoms using coherent Raman drive, which allows us to detect spin coherence in ultracold atoms.

\section{THEORETICAL MODELING}

A pair of phase-coherent Raman radiation fields derived from an external cavity diode laser (ECDL) interacts with a $\Lambda$-type three-level system (3LS) formed by two ground states $|1\rangle,|2\rangle$ and one excited state $|3\rangle$ as depicted in Fig. 1(a) and its inset. The frequency, intensity, and polarization of the Raman fields are controlled using acousto-optic modulators (AOMs) and wave plates.

The semiclassical interaction Hamiltonian of the 3LS with the Raman fields can be written as [36]

$$
\begin{aligned}
\frac{\mathcal{H}}{\hbar}= & \left(\Delta_{23}-\Delta_{13}\right) \mu^{\dagger} \mu-\Delta_{13} \sigma^{\dagger} \sigma-\Omega_{13}\left(\sigma+\sigma^{\dagger}\right) \\
& -\Omega_{23}\left(\mu+\mu^{\dagger}\right),
\end{aligned}
$$

where we define the dipole transition operators of the 3LS by $\sigma^{\dagger}=|1\rangle\left\langle 3\left|, \mu^{\dagger}=\right| 2\right\rangle\left\langle 3\left|, \nu^{\dagger}=\right| 1\right\rangle\langle 2|$. Here, $\Omega_{13}$ and $\Omega_{23}$ are the resonant Rabi angular frequency of the Raman field 1 (R1) and Raman field 2 (R2), respectively. For simplicity, we have assumed $\Omega_{13}$ and $\Omega_{23}$ to be real valued. The detunings of the Raman fields from the related optical transitions are $\Delta_{13}=\omega_{s 1}-\omega_{3}+\omega_{1}, \Delta_{23}=\omega_{s 2}-\omega_{3}+\omega_{2}$, where $\omega_{s 1}\left(\omega_{s 2}\right)$ is the angular frequency of the Raman field 1(2) and $\omega_{i}$ is 


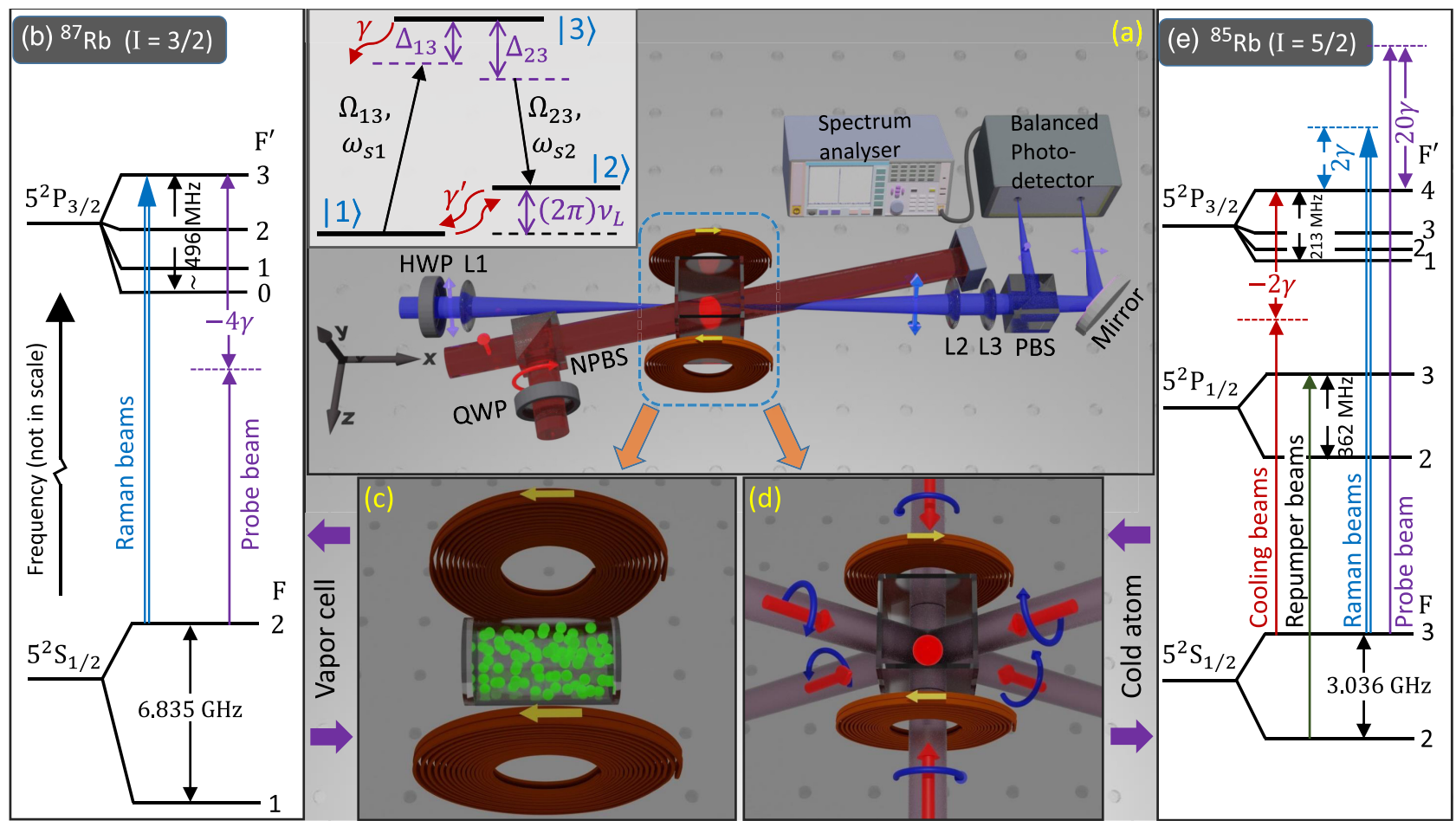

FIG. 1. The schematic of the experimental setup and the relevant energy levels of the rubidium (Rb) atoms presented in this paper. (a) depicts the arrangements of the Raman beams (red), probe beam (blue), magnetic coils, and detection setup. The blue dashed rectangle shows the $\mathrm{Rb}$ atoms in presence of different environments, which is investigated in this paper: (c) thermal ${ }^{87} \mathrm{Rb}$ atoms (with neon buffer gas) in a quartz cell in presence of homogeneous magnetic fields, and (d) in situ magneto-optically trapped (MOT) ${ }^{85} \mathrm{Rb}$ atoms inside an ultrahigh vacuum glass cell in presence of inhomogeneous trapping magnetic fields. The faint beams in (d) are the cooling and repumper laser radiation fields used to capture the ${ }^{85} \mathrm{Rb}$ atoms in a MOT. Here the red arrows and blue circular arrows represent the direction of the laser beams and their polarizations. The red blob at the center of the glass cell represents the cold ${ }^{85} \mathrm{Rb}$ atomic cloud. HWP: half-wave plate, QWP: quarter-wave plate, L1, L2, L3 lenses, NPBS nonpolarizing beam splitter, PBS: polarizing beam splitter. (b) The relevant electronic energy level diagram of ${ }^{87} \mathrm{Rb}$ atom [37] and the probe and Raman laser frequencies for the vapor cell measurements. (e) represents the electronic (dipole) transition lines for ${ }^{85} \mathrm{Rb}$ atom [38] and the frequencies of the cooling and repumper, Raman, and the probe beams. I is the nuclear spin, $F\left(F^{\prime}\right)$ represents the ground (excited) hyperfine levels, and $\gamma$ is the linewidth of the $F^{\prime}$-levels of the respective atoms. (a) Inset: Energy level diagram used for coherent coupling between the Zeeman states $(|1\rangle$ and $|2\rangle)$ within a ground $F$-level. $\Delta_{i 3}, \Omega_{i 3}$, and $\omega_{s i}(i=1,2)$ are the optical detuning, Rabi frequency, and frequencies of the two Raman fields. $\gamma$ is the excited state $\left(|3\rangle\right.$, a Zeeman state in $F^{\prime}-$ level) linewidth, and $\gamma^{\prime}$ is the relaxation rate of spin coherence between the states $|1\rangle$ and $|2\rangle$. The Raman resonance condition is satisfied when the frequency difference between the Raman fields coincides with the frequency difference between the states $|1\rangle$ and $|2\rangle$ (i.e., $\omega_{s 1}-\omega_{s 2}=2 \pi v_{L}$ ), where $v_{L}$ is the Larmor frequency

that of state $|i\rangle(i=1,2,3)$. The symbols $s_{1}, s_{2}$ denote the polarizations (linear or circular) of the two Raman fields.

An off-resonant, linearly polarized probe laser field generated from another ECDL propagating along the $x$ direction dispersively detects the temporal fluctuations of the population between the Zeeman states within a ground hyperfine level of rubidium atoms. The measured instantaneous Faraday rotation of the probe field is proportional to instantaneous population difference between consecutive Zeeman states. Therefore, the autocorrelation of the Faraday rotation signal can be represented by a two-time correlation $\left\langle\tilde{\rho}_{21}^{\dagger}(t) \tilde{\rho}_{21}(0)\right\rangle$ of density matrix coherence $\left(\tilde{\rho}_{21}\right)$ between these states in the laboratory frame. We write the power spectrum by taking the Fourier transform of such a correlation,

$$
P(\omega)=\frac{1}{2 \pi} \int_{-\infty}^{\infty} d t e^{i \omega t}\left\langle\tilde{\rho}_{21}^{\dagger}(t) \tilde{\rho}_{21}(0)\right\rangle
$$

where the expectation is performed over equilibrium thermal noise. We clarify that the experimentally measured power spectrum of the probe laser's Faraday rotation fluctuations is proportional to $P(\omega)$, and the proportionality constant (with a unit) depends on the atom-probe laser coupling parameters (e.g., probe intensity, probe detuning, oscillator strength, and so on). This proportionality constant between the driven and nondriven case remains the same for all measurements and depends only on the atom-probe laser scattering cross section. In the experiments, we have explicitly measured this cross section and can consistently compare the nondriven and coherently driven cases using $P(\omega)$.

In the absence of driving $(\Omega=0)$ by the Raman fields, the intrinsic SN power spectrum for the spontaneous fluctuations of the population in equilibrium is given by (see Appendix B)

$$
\left.P(\omega)\right|_{\Omega=0}=\frac{N_{2}}{2 \pi} \frac{\gamma_{21}}{\gamma_{21}^{2}+\left(\omega-\omega_{2}+\omega_{1}\right)^{2}},
$$

where $\gamma_{i j}$ is the relaxation rate of the spin coherence between states $i$ to $j$ of the atoms and $N_{2}$ is the number of atoms in the observation region. $\left.P(\omega)\right|_{\Omega=0}$ is a Lorentzian centered around $\omega=\left(\omega_{2}-\omega_{1}\right)=: 2 \pi v_{L}$ and with a full width at half maximum (FWHM) of $2 \gamma_{21}$. 
In the presence of the Raman fields, the atoms are driven out of equilibrium and the population of the Zeeman states starts to oscillate coherently with increasing $\Omega_{13}$ and $\Omega_{23}$. For strong driving, the intrinsic fluctuations (related to equilibrium noise) form a broad background in the measured power spectrum. Therefore, we ignore the equilibrium noise in leading order to obtain a simple expression for the power spectrum of the strongly driven atoms at steady state (see Appendix B),

$$
P(\omega)=\delta\left(\omega+\omega_{s 2}-\omega_{s 1}\right)\left|\rho_{21}\right|^{2},
$$

where $\tilde{\rho}_{21}(t)=\rho_{21} e^{i\left(\omega_{s_{2}}-\omega_{s_{1}}\right) t}$. We can find a relatively simple formula for $\rho_{21}$ by choosing $\Omega_{13}=\Omega_{23}=\Omega$ [39], $\gamma_{13}=$ $\gamma_{23}=\gamma, \gamma_{12}=\gamma_{21}=\gamma^{\prime}$, and $\Delta_{23}=0, \Delta_{13}=\Delta$ :

$$
\rho_{21}=-\frac{\gamma \Omega^{2}\left(\left(\gamma^{\prime}+i \Delta\right)(2 \gamma+i \Delta)+4 \Omega^{2}\right)}{\gamma\left(\gamma^{\prime 2}+\Delta^{2}\right)\left(2 \gamma^{2}+\Delta^{2}\right)+\left(2 \gamma \gamma^{\prime}\left(3 \gamma^{\prime}+4 \gamma\right)+\left(\gamma^{\prime}+2 \gamma\right) \Delta^{2}\right) \Omega^{2}+4\left(3 \gamma^{\prime}+2 \gamma\right) \Omega^{4}} .
$$

$P(\omega)$ in Eq. (4) shows a delta peak at $\omega=\omega_{s_{1}}-\omega_{s_{2}}=: 2 \pi \delta_{12}$ [40], whose strength $\left|\rho_{21}\right|^{2}$ in Eq. (5) grows rapidly with increasing $\Omega$ before saturating at large $\Omega$.

\section{ENHANCEMENT OF SIGNAL STRENGTH USING RAMAN DRIVING}

We start by demonstrating the signal-strength enhancement with the Raman driving as compared to the intrinsic SN signal (i.e., $\Omega=0$ ) in the thermal atomic vapor. A vapor cell containing ${ }^{87} \mathrm{Rb}$ atoms and neon buffer gas is placed in a uniform magnetic field (along $\hat{z}$ ) produced by a Helmholtz coil as shown in Fig. 1(c). We select the three states as $\left|F=2, m_{F}=-1\right\rangle \equiv|1\rangle,\left|F=2, m_{F}=0\right\rangle \equiv|2\rangle$, and $\mid F^{\prime}=$ $\left.3, m_{F^{\prime}}=0\right\rangle \equiv|3\rangle$ [see Fig. 1(b) and Fig. 1(a), inset]. We align the Raman fields with a small angle $\sim 3^{\circ}$ with respect to the $x$ axis to prevent them from falling on the photo detector.

The probe laser field is detuned by $-4 \gamma$ [41] from the $5 S_{1 / 2}, F=2 \rightarrow 5 P_{3 / 2}, F^{\prime}=3\left(\mathrm{D}_{2}\right)$ transition of ${ }^{87} \mathrm{Rb}$ as depicted in Fig. 1(b). The measured $\left.P(\omega)\right|_{\Omega=0}$ is presented in Fig. 2(a), which shows the Lorentzian FWHM of $\sim 2 \pi \times$ $150 \mathrm{kHz}$ for ${ }^{87} \mathrm{Rb}$ atoms in thermal equilibrium at $T=388 \mathrm{~K}$. The Faraday rotation is an angle having unit of rad. Since we have performed a polarimetric measurement on a balanced photo detector with an output in volts, the power spectral density has a unit of $\mathrm{V}^{2} / \mathrm{Hz}[24,42]$. The data acquisition time for this measurement is $98 \mathrm{~s}$. The details of the instrumentation as well as the detection scheme using the polarimetric technique and the swept-frequency spectrum analyser used can be found in Refs. [18,43].

In the presence of the Raman fields $(\Omega \neq 0)$, we observe an enhancement of $P(\omega)$, as shown in Fig. 2(b) [44]. The peak height is maximum when $\Delta=0$ (i.e., $\delta_{12}=v_{L}$ ) and it decreases with increasing $|\Delta|$. The envelope of the narrow peaks with varying $\delta_{12}$, as shown in Fig. 2(b), has a Lorentzian-like form when $\Omega<\gamma$. But it switches to a Gaussian-like shape for larger $\Omega$. For the experiments performed in this work, the $\Omega / \gamma$ has always been less than 1 , therefore we explored the Lorentzian-like regime.

In the insets of Fig. 2(b), we show a comparison between the spectrum observed in the Raman-resonance condition for $\Omega / \gamma=7.6 \times 10^{-3}$ (top) and that at $\Omega / \gamma=0$ (bottom). An enhancement by $10^{5}$ in the signal strength $\left(\left|\rho_{21}\right|^{2}\right)$ has been observed for the driven system. In the subsequent experiments, we vary $\delta_{12}$ while keeping $\Delta_{23}=0$, and record a series of spectra shown in Fig. 2(b). These series of spectra span an envelope that is fitted by Eq. (5) (pink dashed line) with fitting parameters $\Omega / \gamma=7.6 \times 10^{-3}$ and $2 \gamma^{\prime}=2 \pi \times 118.6 \mathrm{kHz}$, which gives a peak position at $v_{L}$ and an envelope width of $\sim 2 \pi \times 250 \mathrm{kHz}$. The typical data collection time for an individual spin correlation signal shown in the inset (top panel) of Fig. 2(b) is $1 \mathrm{~s}$.

The dependence of $\left|\rho_{21}\right|^{2}$ on $\Omega$ can be understood by taking the Raman resonant limit $\Delta=0\left(\delta_{12}=v_{L}\right)$ in Eq. (5) and we get

$$
\left|\rho_{21}\right|_{\Delta=0}^{2}=\frac{\gamma^{2} \Omega^{4}}{\left(\gamma^{\prime} \gamma^{2}+\left(3 \gamma^{\prime}+2 \gamma\right) \Omega^{2}\right)^{2}},
$$

which shows that the coherence between the ground levels grows with increasing $\Omega$ before saturating for higher $\Omega>\gamma$. To measure $\left|\rho_{21}\right|_{\Delta=0}^{2}$, we vary the intensities of the Raman fields keeping $\Delta=0$. The polarization of the Raman fields are $\left(\pi_{1}\right)_{x}-\left(\sigma_{2}^{+}\right)_{x}$ for the measurements presented in Figs. 2(b) and Fig. 3, where the subscript 1(2) refers to R1 (R2), and $x$ axis is the propagation direction of the Raman fields. The measured on-resonance peak strength $\left|\rho_{21}\right|_{\Delta=0}^{2}$ as a function of $\Omega / \gamma$ is plotted in Fig. 3(a). The black triangles (blue circles) are the data corresponding to $T=373 \mathrm{~K}(393 \mathrm{~K})$ of the vapor cell. We fit these data by Eq. (6) (solid lines), keeping only $\gamma^{\prime}$ as a free parameter. We extract the value of $2 \gamma^{\prime}$ to be $2 \pi \times(95 \pm 7) \mathrm{kHz}$ and $2 \pi \times(136 \pm 15) \mathrm{kHz}$ for $373 \mathrm{~K}$ and $393 \mathrm{~K}$, respectively. We have separately measured the FWHM of the intrinsic SN spectrum to be $2 \pi \times(126 \pm 3) \mathrm{kHz}$ and $2 \pi \times(153.7 \pm 0.4) \mathrm{kHz}$ for these temperatures, respectively. We attribute these small but finite (within 25\%) differences to two competing effects of different physical origins-the perturbation induced by the Raman driving to bring the atoms beyond thermal equilibrium and linear response, and the suppression of spin projection noise due to coherent coupling.

For a fixed $\Omega$, we vary $\delta_{12}$ and record the envelope of $P(\omega)$. We repeat these experiments for various values of $\Omega / \gamma$ and fit each spectrum with a Lorentzian function. The extracted FWHMs of the envelope for various $\Omega / \gamma$ are shown in Fig. 3(b). Black triangles (blue circles), shown in the inset, are the extracted values of $2 \gamma^{\prime}$ after fitting the envelope with $\left|\rho_{21}\right|^{2}$ given in Eq. (5) for $T=373 \mathrm{~K}(393 \mathrm{~K})$, respectively. The average value of $2 \gamma^{\prime}$, extracted from these measurements are $2 \pi \times(95 \pm 6) \mathrm{kHz}$ and $2 \pi \times(131 \pm 10) \mathrm{kHz}$ for those two temperatures, respectively. In the measurements reported in Fig. 3(b) (inset), we notice consistent lower values of the extracted $2 \gamma^{\prime}$ than the intrinsic measurements $(\Omega=0)$, which indicates that the spin projection noise suppression is more 

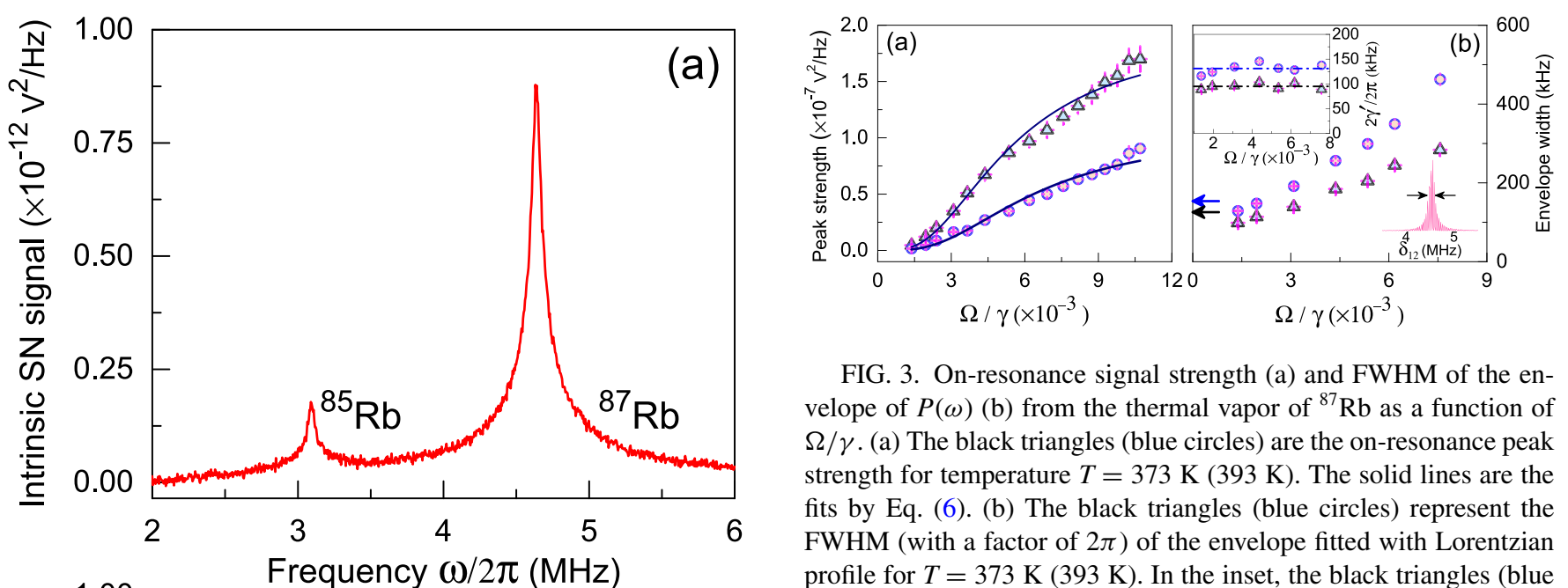

FIG. 3. On-resonance signal strength (a) and FWHM of the envelope of $P(\omega)$ (b) from the thermal vapor of ${ }^{87} \mathrm{Rb}$ as a function of $\Omega / \gamma$. (a) The black triangles (blue circles) are the on-resonance peak strength for temperature $T=373 \mathrm{~K}(393 \mathrm{~K})$. The solid lines are the fits by Eq. (6). (b) The black triangles (blue circles) represent the FWHM (with a factor of $2 \pi$ ) of the envelope fitted with Lorentzian profile for $T=373 \mathrm{~K}(393 \mathrm{~K})$. In the inset, the black triangles (blue circles) show the extracted value of $2 \gamma^{\prime}$ after fitting the envelope using Eq. (5). The black (blue) arrow indicated in the bottom-left side represents the measured FWHM of the intrinsic SN spectrum. The raw spectrum and its measured FWHM are indicated at the bottom-right corner.

ments of Faraday rotation have been demonstrated in cold atomic systems in the recent past [11,45-48]. Here, we apply the Faraday rotation technique to study spin coherence produced by a coherent Raman excitation. Our experimental system typically traps more than $10^{7}$ atoms at a temperature of $150 \pm 10 \mu \mathrm{K}$ in a standard vapor loaded MOT with a typical Gaussian width of $\sim 4 \mathrm{~mm}$. We take sufficient care to ensure that the center of the atomic cloud is overlapped with the zero of the quadrupole trapping field within $30 \mu \mathrm{m}$ [see Fig. 1(d)]. More details about the experimental system are provided in Appendix A. Additionally, we align a pair of Raman fields with a waist diameter of $6 \mathrm{~mm}$, blue detuned by $2 \gamma(\gamma=$ $2 \pi \times 6.1 \mathrm{MHz})$ from $5 S_{1 / 2}, F=3 \rightarrow 5 P_{3 / 2}, F^{\prime}=4\left(\mathrm{D}_{2}\right)$ transition. A collimated probe laser field along $\hat{x}$ with a waist diameter $70 \mu \mathrm{m}$ and blue detuned by $20 \gamma$ from the same transition is sent through the cold atomic cloud [see Figs. 1(a) and 1(e)]. The probe field position with respect to the cloud center is independently measured using high precision absorption spectroscopy and absorption imaging within a precision of 10 $\mu \mathrm{m}$. The quadrupole magnetic field profile creating the MOT is separately characterized using a Hall magnetometer probe, and it is shown schematically in Fig. 4(b).

To measure $P(\omega)$ at a finite Larmor frequency in the presence of the Raman fields, we have shifted the probe laser by $900 \mu \mathrm{m}$ from the trap center in the $z$ direction on the $x-z$ plane. Also, due to the Raman fields, the cloud center shifts in the $x$ direction depending on the Raman fields intensity and the detuning [see Fig. 4(b)]. In Fig. 4(a), we show a representative spectrum detected in this condition at $\delta_{12}=2.73 \mathrm{MHz}$ for $\Omega / \gamma=0.35$. In the absence of the Raman fields-unlike in the vapor case-we do not observe any detectable intrinsic $\mathrm{SN}$ signal due to the fact that the total number of atoms within the probe field is $10^{4}$ times less than that of thermal vapor.

We record a series of $P(\omega)$ by varying $\delta_{12}$. For a fixed value of $\Omega / \gamma$ and probe field position, we obtain a composite spectra [see Fig. 4(c)] by repeating the above procedure and recording the peak height of each individual $P(\omega)$. 

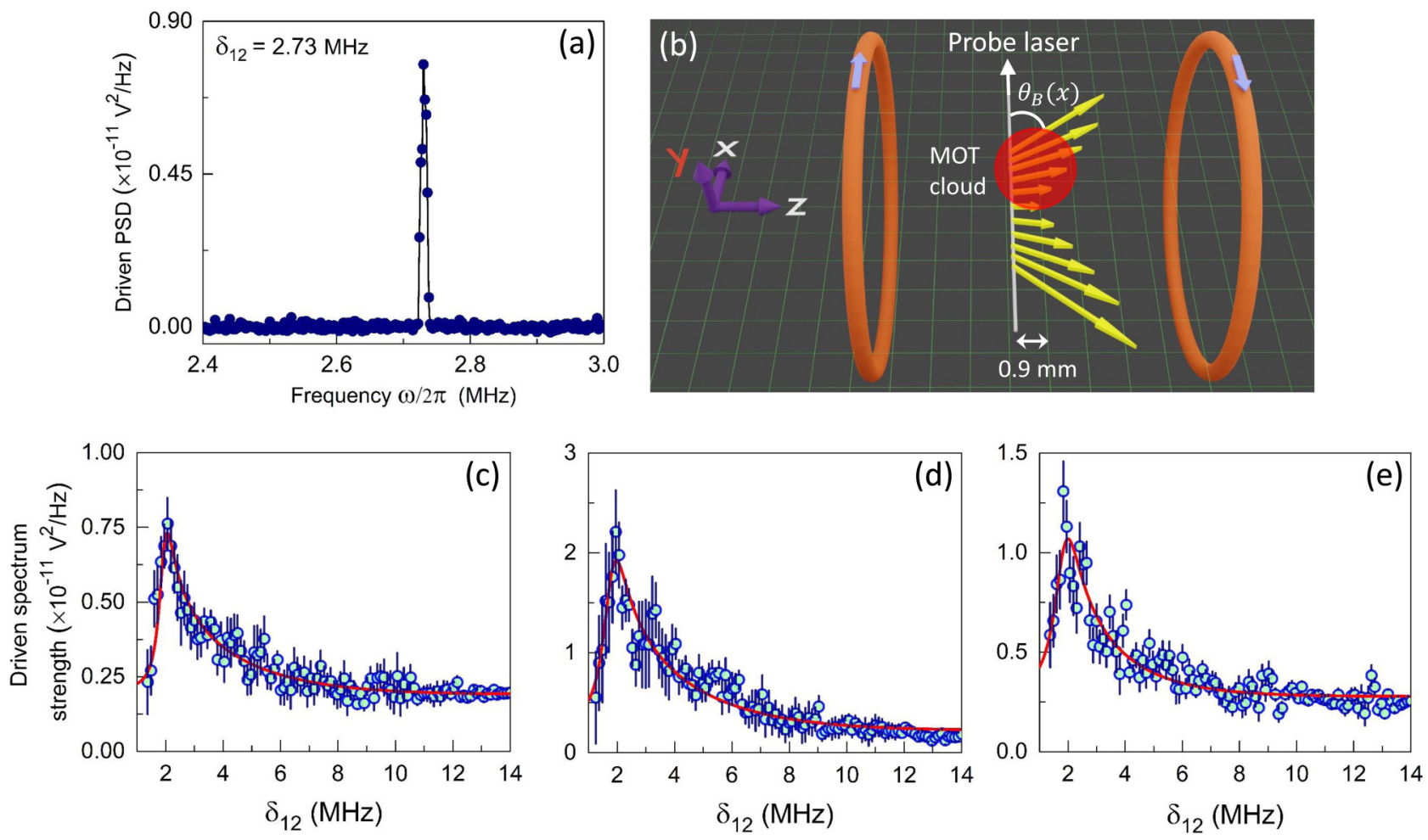

FIG. 4. Measurement of spin coherence in Raman-driven cold ${ }^{85} \mathrm{Rb}$ atoms at $150 \mu \mathrm{K}$. (a) $P(\omega)$ recorded at $\delta_{12}=2.73 \mathrm{MHz}$. (b) A schematic representation of our experiment including local magnetic field profiles (yellow arrows), the position of the cold atomic cloud (red blob), and the probe laser. (c)-(e) The recorded envelope spectra (blue open circles) for the various sets (check the text for details) of experimental parameters.

The acquisition time for each data point is $8 \mathrm{~s}$ for a signal run of the experiment. We analyze our experimental results using the earlier modeling for thermal vapors after including corrections due to magnetic field variations, atom density distributions, and multilevel contributions (see Appendix C). The asymmetric nature of the spectrum in Fig. 4(c) arises mostly due to the magnetic field variations within the trapped MOT cloud and the Gaussian atom density distributions of the cloud. However, for the probe beam position $|z|<1 \mathrm{~mm}$ on the $x-z$ plane as in the case shown in Fig. 4(c), the magnetic field orientation $\left[\theta_{B}(x)\right]$ with respect to the $z$ axis is the most dominating factor for the asymmetric nature of the envelope spectrum over the Gaussian atom density distribution of the MOT. The multilevel corrections have a negligibly small contribution in this asymmetric nature. We fit the data in Fig. 4(c) (solid red line) with a free parameter $\gamma^{\prime}$. We get an estimate for the value of $2 \gamma^{\prime}$ from this fitting as $2 \gamma^{\prime}=$ $2 \pi \times(1.0 \pm 0.7) \mathrm{kHz}$.

We have repeated the above measurements for various $z$ positions of the probe laser. Another representative data is shown in Fig. 4(d) for $z=700 \mu \mathrm{m}$, which gives $2 \gamma^{\prime}=$ $2 \pi \times(2.3 \pm 0.7) \mathrm{kHz}$. We have also performed the experiment in the presence of balanced Raman fields, which minimize the shift of the cloud center as shown in Fig. 4(e). The extracted value of $2 \gamma^{\prime}$ is $2 \pi \times(1.3 \pm 0.7) \mathrm{kHz}$. Our present experiment is limited by several factors, e.g., magnetic field inhomogeneity, off-resonant scattering, the effect of strong driving fields, and relative frequency stability of the Raman fields, which can significantly change the measured spin relaxation rate. At this low temperature $(150 \mu \mathrm{K})$, the above perturbation effects are typically far more important than the suppression of spin projection noise discussed earlier. Nevertheless, the observed reduction in extracted spin relaxation rate by two orders of magnitude as compared to the thermal atoms, which can be attributed to the six-order lowering of the temperature that substantially reduces thermal coupling, collisions, and transit times.

\section{DEPENDENCE OF DRIVEN POWER SPECTRUM ON RAMAN FIELDS' POLARIZATION}

We have experimentally investigated the dependence of the Raman-driven power spectrum on the polarization state of the Raman fields in both thermal vapors and cold atomic clouds.

In Fig. 5(a), we show the driven power spectrum for various combinations of the polarization state of the Raman fields in thermal vapors. The polarization of the $\mathrm{R} 1$ field is linear $\left[\left(\pi_{1}\right)_{x}\right]$ and kept fixed. We have tuned the polarization state of the R2 field and recorded the driven power spectrum as shown in Fig. 5(a). The strength of the spectrum is maximum for $\left(\pi_{1}\right)_{x}-\left(\sigma_{2}^{+}\right)_{x}$ polarization of R1 and R2 fields, which corresponds to the angle $\theta=45^{\circ}$ or $225^{\circ}$ between the optic axis of the quarter-wave plate (QWP) and the input polarization ( $p$ polarized) of the R2 field. The Raman fields cannot drive the atoms coherently between states $|1\rangle$ and $|2\rangle$ for polarization combination $\left(\pi_{1}\right)_{x}-\left(\pi_{2}\right)_{x}$, which corresponds to $\theta=90^{\circ}$ or $180^{\circ}$. This fact was experimentally confirmed and is presented in Fig. 5(a), which shows that we indeed coherently drive the entire atomic sample as opposed to incoherent driving. Note that we have observed an additional maximum at 

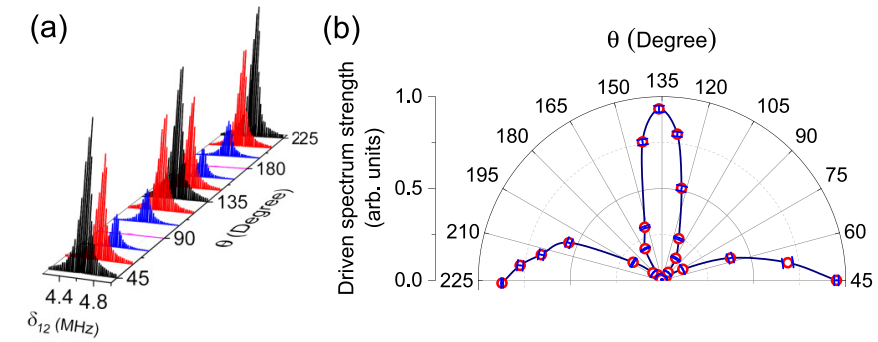

FIG. 5. (a) The Raman-driven power spectrum with detuning $\delta_{12}$ for various polarization states of the $\mathrm{R} 2$ field in thermal vapors. The $\mathrm{R} 1$ field is $p$ polarized $\left[\left(\pi_{1}\right)_{x}\right] . \theta$ is the angle between the optic axis of the quarter-wave plate (QWP) and the input polarization ( $p$ polarized) of the R2 field. The angular momentum conservation of light-matter interactions in $\Lambda$ system is satisfied for $\left(\pi_{1}\right)_{x}-\left(\sigma_{2}^{ \pm}\right)_{x}$ polarization combinations of the Raman fields. These correspond to $\theta=45^{\circ}, 135^{\circ}, 225^{\circ}$, where a maximum in the spectrum is observed. The driven spectrum vanishes for $\left(\pi_{1}\right)_{x}-\left(\pi_{2}\right)_{x}$ polarization combinations $\left(\theta=90^{\circ}\right.$ and $\left.180^{\circ}\right)$, implying no coherent coupling between the states $|1\rangle \leftrightarrow|2\rangle$ by the Raman fields. (b) The driven spectrum strength as a function of $\theta$ at Raman resonance condition. The solid lines joining the data points are a guide to the eye.

$\theta=135^{\circ}$. The appearance of this maximum can be explained in the above way by considering a $\Lambda$ system with ground states $\left|F=2, m_{F}=-1\right\rangle \equiv|1\rangle,\left|F=2, m_{F}=0\right\rangle \equiv|2\rangle$, and excited state $\left|F^{\prime}=3, m_{F^{\prime}}=-1\right\rangle \equiv|3\rangle$. Such a combination of states is allowed for the alkali atom ${ }^{87} \mathrm{Rb}$ in our thermal vapor experiments.

We here demonstrate the role of angular momentum conservation in coherent coupling through our measurements. In Fig. 5(b), the normalized peak strength of the driven power spectrum $\left(\left|\rho_{21}\right|_{\Delta=0}^{2}\right)$ from thermal vapors is shown for various angle $\theta$. The observation in Fig. 5(b) shows the fidelity of the coherent coupling of atoms by the Raman fields' polarization state. This can also be applied to control the atomic coherence between ground levels. The manipulation of atomic level coherence may find applications in quantum communications and quantum information processing using neutral atoms.

A similar study for the polarization dependence of the driven power spectrum in cold rubidium atoms is shown in Fig. 6. We have fixed $\delta_{12}=2.73 \mathrm{MHz}$ to resonantly drive the atoms located at $x=1.9 \mathrm{~mm}$, and $z=900 \mu \mathrm{m}$ where $\theta_{B} \approx 45^{\circ}$. The dependence of $P(\omega)$ on the Raman fields' polarization for cold atoms is similar to the previous case with thermal vapors (shown in Fig. 5). While the peak signal strength of $P(\omega)$ reduces exactly to zero at $90^{\circ}$ and $180^{\circ}$ as expected, the magnitude at $\theta=135^{\circ}$ reaches only half of the observed values at $\theta=45^{\circ}$ and $225^{\circ}$ in cold atoms inside the MOT, which is very different from the thermal vapors in homogeneous magnetic field.

\section{A. Raman coherence in the quantization basis}

The experiments in vapor cells were performed in the presence of a homogeneous magnetic field (defining the quantization axis) applied along $\hat{z}$ and the Raman fields propagating along $\hat{x}$. The probe laser propagating along the $x$ direction detects the $x$ component of the atomic spins. Here

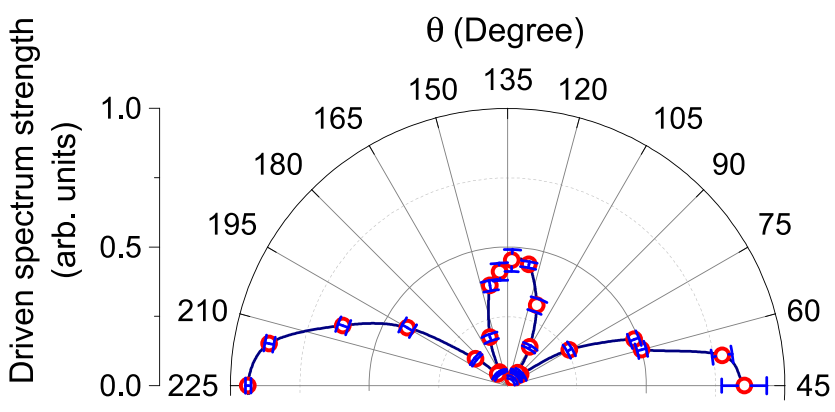

FIG. 6. The driven power spectrum strength at $\delta_{12}=2.73 \mathrm{MHz}$ for various angles $\theta$ in cold atoms. The signal strength at $\theta=135^{\circ}$ is about $1 / 2$ of that at $\theta=45^{\circ}$ and $225^{\circ}$. The polarization sensitivity at each $\delta_{12}$ carries its unique signature for atoms in the MOT, unlike atoms in a homogeneous magnetic field. The solid lines joining the data points are a guide to the eye.

we neglect the slight angle between the probe and the Raman lasers, as schematically shown in the Fig. 7.

We consider the polarization state of the Raman fields propagating along $\hat{k} \| \hat{x}$ being linear $\left[\left(\pi_{1}\right)_{x}\right]$ and circular $\left[\left(\sigma_{2}^{+}\right)_{x}\right]$. In the presence of the Raman fields, the electronic spins align along the $x$ axis. However, due to the homogeneous magnetic field along $\hat{z}$, the spins precess about the $z$ axis on the $x-y$ plane. Since the Larmor precession rate (e.g., $v_{L} \sim$ 4.6 MHz) in our experiments is typically much higher than the spin relaxation rate $\left(1 / 2 \pi T_{2} \sim 0.15 \mathrm{MHz}\right)$, the spins lie on the $x-y$ plane.

We further restrict our discussion for a system with ground hyperfine level $F=1$ and excited hyperfine level $F^{\prime}=1$. In the following, we will describe the dependence of the Raman-driven power spectrum strength on various polarization combinations of R1 and R2 fields presented in Fig. 5. Any spin component of $F$ or $F^{\prime}$ on the $x-y$ plane can be written as a linear superposition of all possible spin components along $\hat{z}$, for an example [49],

$\left|m_{F}=1\right\rangle_{x}=\frac{1}{2}\left|m_{F}=-1\right\rangle_{z}+\frac{1}{\sqrt{2}}\left|m_{F}=0\right\rangle_{z}+\frac{1}{2}\left|m_{F}=1\right\rangle_{z}$.

We can also decompose the polarization of the Raman fields in terms of their electric fields in the following fashion [50,51]:

$$
\left(\pi_{1}\right)_{x} \equiv \hat{e}_{1 y}=\frac{1}{\sqrt{2}}\left(\frac{\hat{e}_{1 y}+i \hat{e}_{1 x}}{\sqrt{2}}\right)+\frac{1}{\sqrt{2}}\left(\frac{\hat{e}_{1 y}-i \hat{e}_{1 x}}{\sqrt{2}}\right),
$$

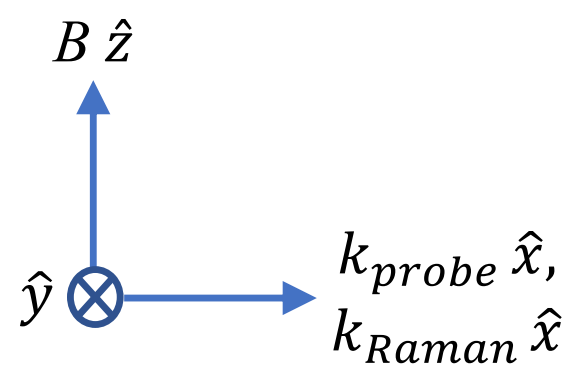

FIG. 7. The diagram depicts the direction of the applied uniform magnetic field $(B \hat{z})$, the propagation direction of probe $\left(k_{\text {probe }} \hat{x}\right)$, and $\operatorname{Raman}\left(k_{\mathrm{Raman}} \hat{x}\right)$ lasers. 


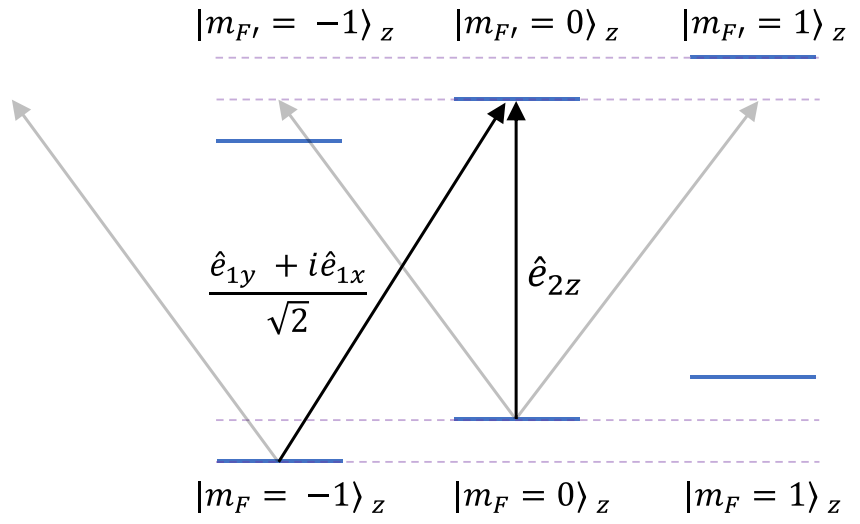

FIG. 8. Formation of a $\Lambda$ system in $\hat{z}$ basis within the Zeeman states for $\left(\pi_{1}\right)_{x}$ and $\left(\sigma_{2}^{+}\right)_{x}$ combination of polarization of the Raman fields. The generated $\Lambda$ system is shown by black arrows.

and

$$
\begin{aligned}
\left(\sigma_{2}^{+}\right)_{x} \equiv & \frac{\hat{e}_{2 z}+i \hat{e}_{2 y}}{\sqrt{2}}=\frac{\hat{e}_{2 z}}{\sqrt{2}}+\frac{i}{2}\left(\frac{\hat{e}_{2 y}+i \hat{e}_{2 x}}{\sqrt{2}}\right) \\
& +\frac{i}{2}\left(\frac{\hat{e}_{2 y}-i \hat{e}_{2 x}}{\sqrt{2}}\right),
\end{aligned}
$$

where $\hat{e}_{1 i}$ or $\hat{e}_{2 i}$ is the $i$ th component of the corresponding electric field with $i=x, y, z$.

In our experiment, we fix the frequency of the R1 field [of $\left(\pi_{1}\right)_{x}$ polarization] on resonance to $\left|m_{F}=-1\right\rangle_{z} \leftrightarrow \mid m_{F^{\prime}}=$ $0\rangle_{z}$ transition, and that of the $\mathrm{R} 2$ field [of $\left(\sigma_{2}^{+}\right)_{x}$ polarization] on resonance to the $\left|m_{F}=0\right\rangle_{z} \leftrightarrow\left|m_{F^{\prime}}=0\right\rangle_{z}$ transition. According to our decomposition in Eqs. (8) and (9), the allowed optical transitions in the $\hat{z}$ basis can be shown in Fig. 8, where the field $\hat{e}_{2 z}$ couples $\left|m_{F}=0\right\rangle_{z} \leftrightarrow\left|m_{F^{\prime}}=0\right\rangle_{z}$ transitions and the field $\left(\hat{e}_{1 y}+i \hat{e}_{1 x}\right) / \sqrt{2}$ couples $\left|m_{F}=-1\right\rangle_{z} \leftrightarrow\left|m_{F^{\prime}}=0\right\rangle_{z}$ transitions.

Figure 8 shows that a $\Lambda$-type $3 \mathrm{LS}$ is formed (indicated by black arrows) in the $\hat{z}$ basis, and a coherence is built between the states $\left|m_{F}=-1\right\rangle_{z} \leftrightarrow\left|m_{F}=0\right\rangle_{z}$. The coherence between $\left|m_{F}=0\right\rangle_{z}$ and $\left|m_{F}=1\right\rangle_{z}$ can also be explained in a similar fashion. This coherence in $\hat{z}$ basis in turn built a coherence in the $\hat{x}$ basis via Eq. (7), and detected by the off-resonant probe laser. This case corresponds to $\theta=45^{\circ}$ and $225^{\circ}$ in Fig. 5 . The other maxima at $\theta=135^{\circ}$ can be explained by considering the $\left(\pi_{1}\right)_{x}$ and $\left(\sigma_{2}^{-}\right)_{x}$ combination of the polarization states of the R1 and R2 field.

For $\left(\pi_{1}\right)_{x}-\left(\pi_{2}\right)_{x}$ combination of R1 and R2 field polarizations $\left(\theta=90^{\circ}\right.$ and $\left.180^{\circ}\right)$, no $\Lambda$ system is formed in $\hat{z}$ basis within the ground hyperfine level Zeeman states. Therefore, no amplification in the driven power spectrum has been observed.

TABLE I. Dependence of Raman-driven power spectrum signal strength on the Raman fields' polarization combination.

\begin{tabular}{lc}
\hline \hline Polarization of Raman fields & Comments on signal strength \\
\hline$\left(\pi_{1}\right)_{x}-\left(\pi_{2}\right)_{x}$ and $\left(\sigma_{1}^{ \pm}\right)_{x}-\left(\sigma_{2}^{\mp}\right)_{x}$ & No amplification, intrinsic \\
$\left(\pi_{1}\right)_{x}-\left(\sigma_{2}^{ \pm}\right)_{x}$ and $\left(\sigma_{1}^{ \pm}\right)_{x}-\left(\pi_{2}\right)_{x}$ & $\left|\rho_{21}\right|^{2}$ \\
$\left(\sigma_{1}^{ \pm}\right)_{x}-\left(\sigma_{2}^{ \pm}\right)_{x}$ & $2\left|\rho_{21}\right|^{2}$ \\
\hline \hline
\end{tabular}

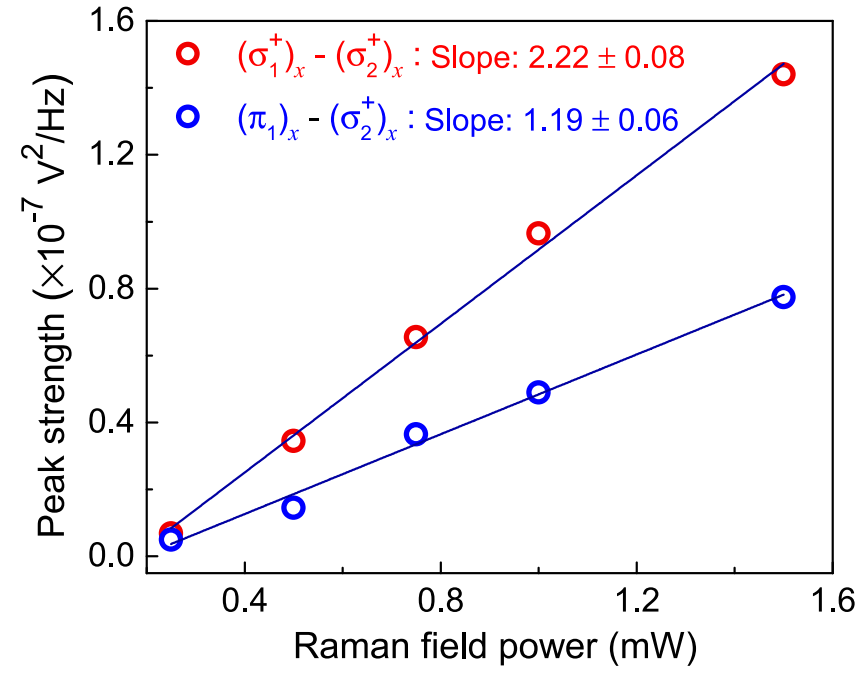

FIG. 9. The on-resonance signal strength of the Raman driven spectrum for $\left(\sigma_{1}^{+}\right)_{x}-\left(\sigma_{2}^{+}\right)_{x}$ and $\left(\pi_{1}\right)_{x}-\left(\sigma_{2}^{+}\right)_{x}$ combination of the Raman fields' polarization with various driving intensities. The plots support the results summarized in Table I.

However, it can be shown using Eq. (9) that when both the Raman fields are $\sigma^{+}$polarized, a double $\Lambda$ system is formed within the consecutive Zeeman states in $F$ manifolds. In this case, the signal strength is two times stronger than the case discussed in Fig. 8.

Table I summarizes the Raman driven signal strength for various combinations of the Raman fields' polarizations.

We have experimentally verified the dependence of the on-resonance Faraday rotation fluctuation signal strength on different polarization combinations of the Raman fields. The experimental results are shown in Fig. 9 for completeness.

\section{CONCLUSIONS AND OUTLOOK}

We demonstrate an enhancement of the Faraday rotation fluctuation signal by five orders of magnitude in thermal rubidium atoms driven by coherent Raman radiation fields. Utilizing this technique, we report relatively noninvasive detection of spin coherence in cold atoms. We also show in detail the dependence of the spin coherence on Raman fields' polarization state.

This paper can further be extended to study spin coherence in cold atomic ensembles confined in a far-off-resonant optical dipole trap. This would avoid the presence of an inhomogeneous magnetic field. Additionally, a free-falling cold atomic cloud provides a clean system unconfined by the trapping magnetic and quasiresonant optical fields. The measurement of the transverse spin coherence in such a system can be performed by applying a homogeneous magnetic field along a preferred direction. However, the data acquisition time for such a measurement is limited by few tens of milliseconds and requires a high temporal resolution detection setup to probe the signal. In the future, such a measurement can be performed using, for example, our recently developed timeresolved digital receiver system [43].

The detection method developed and described in this paper can have significant applications in precision magne- 
tometry, high-resolution imaging, nonperturbative probing of quantum phase transitions in cold atoms, and other similar systems such as cold ions and cold molecules.

\section{ACKNOWLEDGMENTS}

This work was partially supported by the Ministry of Electronics and Information Technology (MeitY), Government of India, through the Center for Excellence in Quantum Technology under Grant No. 4(7)/2020-ITEA. S.R. acknowledges funding from the Department of Science and Technology, India, via the WOS-A Project Grant No. SR/WOS-A/PM59/2019. We acknowledge Hema Ramachandran, Fabien Bretenaker, Priyanka G.L., Meena M.S., Sagar Sutradhar, Snehal Dalvi, and the RRI workshop for instruments, discussions, and technical assistance.

\section{APPENDIX}

This Appendix provides details of our experimental set-up, theoretical modeling, and data analysis methods.

\section{APPENDIX A: DETAILS OF THE COLD ATOM EXPERIMENTAL SETUP}

In the present paper, we have reported the results from two sets of experiments: one in a thermal atomic vapor to develop and characterize the measurement of Faraday rotation fluctuation signals from a coherently driven system and another to perform the main study of the extraction of spin relaxation rate from a cloud of cold ${ }^{85} \mathrm{Rb}$ atoms. Here, we provide details of the second setup. We magneto-optically trap neutral ${ }^{85} \mathrm{Rb}$ atoms inside a glass cell maintaining background pressure less than $10^{-10}$ mbar using standard laser cooling and trapping techniques [see Fig. 1(d) in the main text]. The cooling beams were generated from an ECDL and frequency stabilized to $12 \mathrm{MHz}$ red detuned with respect to $5 S_{1 / 2}, F=$ $3 \rightarrow 5 P_{3 / 2}, F^{\prime}=4\left(\mathrm{D}_{2}\right)$ transitions. The repumping laser beams were derived from another ECDL and frequency stabilized to the $5 S_{1 / 2}, F=2 \rightarrow 5 P_{1 / 2}, F^{\prime}=3\left(\mathrm{D}_{1}\right)$ transition [see Fig. 1(e) in the main text]. A pair of magnetic coils in a near-ideal anti-Helmholtz configuration produces the required spatial magnetic field gradient. We coincided the optical and magnetic field centers with the center of the vacuum chamber with an accuracy of $\sim 30 \mu \mathrm{m}$. We use three independent detection techniques: absorption imaging, fluorescence imaging, and probe absorption for characterizing the cold atoms and alignment of the optical fields for the experiments.

In Fig. 10, we show a typical absorption image of our cold cloud, which gives a good measurement of the total number and spatial distribution of the atoms. Using a separate time-offlight measurement, we obtain the temperature of the atomic cloud to be $\sim 150 \mu \mathrm{K}$. We typically trap more than $10^{7}$ atoms with a Gaussian FWHM of $\sim 4 \mathrm{~mm}$.

The probe laser field is generated from yet another ECDL, and its frequency is monitored using a high-precision wavelength meter (Highfinesse, WSU2) with an absolute frequency accuracy of $1 \mathrm{MHz}$. The collimated probe beam with a Gaussian waist diameter of $70 \mu \mathrm{m}$ was sent through the atomic

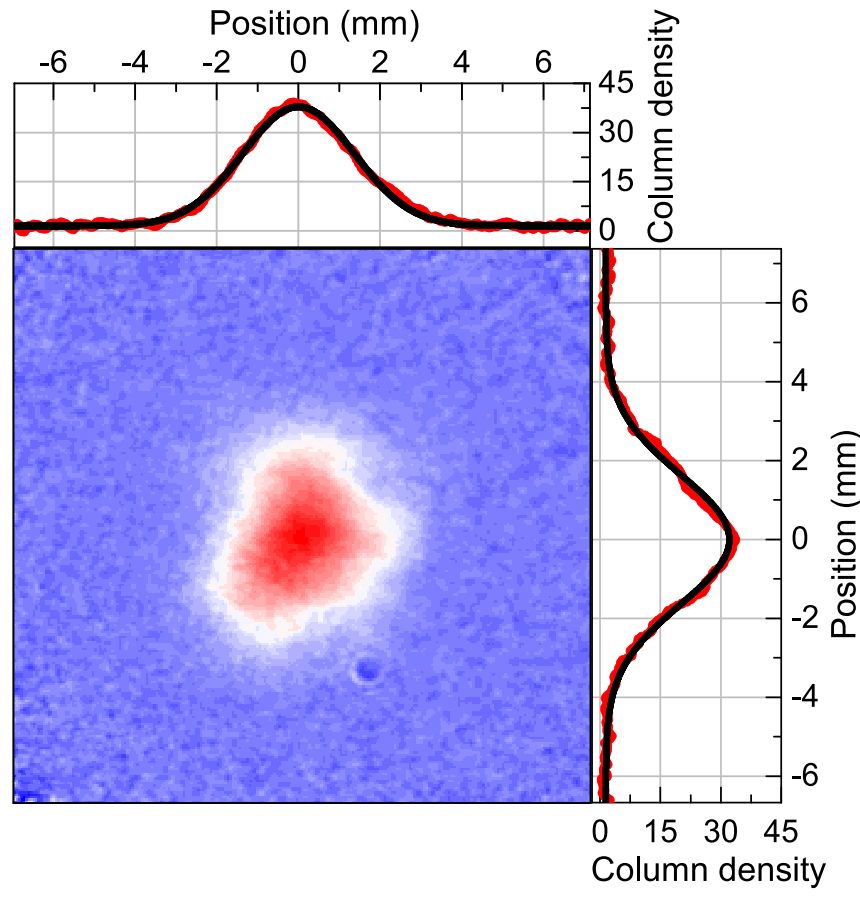

FIG. 10. The absorption image of magneto-optically trapped (MOT) cold ${ }^{85} \mathrm{Rb}$ atomic cloud. The top and right insets show the column density profiles (red) and fit to Gaussian (black) of the trapped cloud.

cloud along $\hat{x}$ at $y=0$ and the $z$ position of the probe beam was varied for different sets of measurements.

The strength and orientation of the magnetic field $B$ vary within the atomic cloud along the probe field direction ( $x$ axis) as shown in Fig. 11. In this configuration, the magnetic field exists only on the $x-z$ plane and we calculate the magnetic field at each position using a solution of the elliptic equations [52]. We separately measure the field components $\left(B_{x}, B_{y}, B_{z}\right)$ using a Hall probe magnetometer (LakeShore) for comparison and calibration purposes. The angle $\theta_{B}(x)$ between the local magnetic field and probe laser propagation direction is position dependent.

The Raman radiation fields for coherent driving were derived from the same ECDL, which provides the cooling laser light. Two independent AOMs were applied to produce the Raman fields with controllable frequency difference (denoted as $\delta_{12}$ in the main text). The radio frequency signal sent into

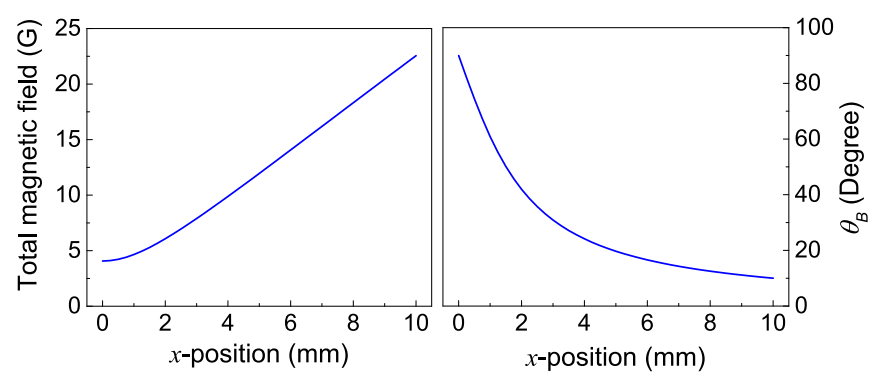

FIG. 11. The $x$ dependence of the total magnetic field and the angle $\theta_{B}(x)$ between the local magnetic field and the probe laser propagation at $y=0$ and $z=900 \mu \mathrm{m}$. 
the AOMs can be tuned using two voltage-controlled crystal oscillators. The control voltage was sent using an Field Programmable Gate Array board with a vertical resolution of 12 bits. The long-term relative frequency jitter of $\delta_{12}$ was measured to be $\sim 7 \mathrm{kHz}$ (for measurement duration of $8 \mathrm{~s}$ ). This jitter limits our measurement precision, which we wish to reduce to a few-Hz level using ultrastable reference sources in our future upgrading of the experimental setup. However, this relative frequency stability is adequate for the first sets of measurements of the Faraday rotation fluctuation signals from the cold atoms. The Raman fields were spatially mode cleaned using PM fibers, expanded to a Gaussian waist diameter of $6 \mathrm{~mm}$ and combined in a nonpolarizing cube beam splitter (NPBS) before sending through the cold atomic cloud. The polarization of the Raman fields can be independently varied, employing the combination of half-wave plates (HWPs) and QWPs. We typically manage to obtain a polarization purity of the Raman fields > 99\%.

\section{APPENDIX B: DETAILS OF THE THEORETICAL MODELING}

\section{Intrinsic spin noise spectrum}

In the absence of driving by the Raman fields $(\Omega=0)$, the rubidium atoms are in thermal equilibrium and the populations in their hyperfine ground level Zeeman states fluctuate over time due to thermal (and quantum) fluctuations. Such equilibrium population fluctuations generate an intrinsic SN in the atomic vapor. When $\Omega=0$, only the lower two levels of the 3LS participate in the equilibrium spin dynamics detected by the probe beam. Therefore, we can derive the SN spectrum by including a noise term in the master equations of the density matrix elements of these levels. Thus, we write

$$
\frac{d \tilde{\rho}_{21}}{d t}=i\left(\omega_{2}-\omega_{1}+i \gamma_{21}\right) \tilde{\rho}_{21}+\eta(t),
$$

where we assume the noise $\eta(t)$ to be a Gaussian white noise with zero mean and $\left\langle\eta\left(t^{\prime}\right) \eta\left(t^{\prime \prime}\right)\right\rangle=N_{2} \gamma_{21} \delta\left(t^{\prime}-t^{\prime \prime}\right)$, where $N_{2}$ is number of atoms within the measurement region. We then get the power spectrum $\left.P(\omega)\right|_{\Omega=0}$ of the spontaneous spin fluctuations as

$$
\begin{aligned}
\left.P(\omega)\right|_{\Omega=0} & =\frac{1}{2 \pi} \int_{-\infty}^{\infty} d t e^{i \omega t}\left\langle\tilde{\rho}_{21}^{\dagger}(t) \tilde{\rho}_{21}(0)\right\rangle \\
& =\frac{1}{\pi} \operatorname{Re}\left[\int_{0}^{\infty} d t e^{i \omega t}\left\langle\tilde{\rho}_{21}^{\dagger}(t) \tilde{\rho}_{21}(0)\right\rangle\right] \\
& =\frac{N_{2}}{2 \pi} \frac{\gamma_{21}}{\gamma_{21}^{2}+\left(\omega-\omega_{2}+\omega_{1}\right)^{2}},
\end{aligned}
$$

where $\omega$ is the spectral frequency.

\section{Raman-driven power spectrum}

In the presence of the Raman fields, the atoms are driven out of thermal equilibrium. We again write phenomenological master equations for the evolution of various components of the density matrix of the 3LS for the Hamiltonian $\mathcal{H}$ in the main text. Since the intrinsic fluctuations [related to the equilibrium noise, e.g., $\eta(t)$ above] only form a broad background in the measured power spectrum for a relatively strong driving by the Raman fields, we drop these noise terms from the following master equations to be able to extract an analytical expression for the measured power spectrum. These master equations are written in the rotating frame by rewriting the elements of coherence as $\rho_{31}(t)=\tilde{\rho}_{31}(t) e^{i \omega_{s_{1}} t}, \rho_{32}(t)=$ $\tilde{\rho}_{32}(t) e^{i \omega_{s_{2}} t}, \rho_{21}(t)=\tilde{\rho}_{21}(t) e^{i\left(\omega_{s_{1}}-\omega_{s_{2}}\right) t}$, where $\tilde{\rho}_{31}(t), \tilde{\rho}_{32}(t)$, and $\tilde{\rho}_{21}(t)$ are the density matrix elements in the laboratory frame and $\rho_{i j}$ are those in the rotated frame. We further take the following limits for the relaxation rates, $\gamma_{13}=\gamma_{23}=$ $\gamma, \gamma_{12}=\gamma_{21}=\gamma^{\prime}, \gamma_{31}=\gamma_{32}=0$, and $\gamma^{\prime} \ll \gamma$, to simplify the master equations:

$$
\begin{gathered}
\frac{d \rho_{11}}{d t}=\gamma\left(1-\rho_{11}-\rho_{22}\right)-i \Omega_{13}\left(\rho_{13}-\rho_{31}\right), \\
\frac{d \rho_{22}}{d t}=\gamma\left(1-\rho_{11}-\rho_{22}\right)-i \Omega_{23}\left(\rho_{23}-\rho_{32}\right), \\
\frac{d \rho_{13}}{d t}=-\left(\gamma+i \Delta_{13}\right) \rho_{13}-i \Omega_{23} \rho_{12} \\
-i \Omega_{13}\left(2 \rho_{11}-1+\rho_{22}\right), \\
\frac{d \rho_{31}}{d t}=-\left(\gamma-i \Delta_{13}\right) \rho_{31}+i \Omega_{23} \rho_{21} \\
+i \Omega_{13}\left(2 \rho_{11}-1+\rho_{22}\right), \\
\frac{d \rho_{23}}{d t}=-\left(\gamma+i \Delta_{23}\right) \rho_{23}-i \Omega_{13} \rho_{21} \\
-i \Omega_{23}\left(2 \rho_{22}-1+\rho_{11}\right), \\
+i \Omega_{23} \rho_{31} . \\
+i \Omega_{23}\left(2 \rho_{22}-1+\rho_{11}\right), \\
\frac{d \rho_{32}}{d t}=-\left(\gamma-i \Delta_{23}\right) \rho_{32}+i \Omega_{13} \rho_{12} \\
\frac{d \rho_{21}}{d t}=-i\left(\Delta_{13}-\Delta_{23}-i \gamma^{\prime}\right) \rho_{12}+i \Omega_{13} \rho_{32} \\
-i \Omega_{23} \rho_{13}, \\
\left.\Delta_{13}-\Delta_{23}+i \gamma^{\prime}\right) \rho_{21}-i \Omega_{13} \rho_{23} \\
+
\end{gathered}
$$

We apply these equations to investigate how the Raman fields affect the coherence $\tilde{\rho}_{21}(t)$ between the ground levels. From the above set of equations, we find $\rho_{21}(t)$ at the steady state by setting $d \rho_{i j}(t) / d t=0$. Since we ignore the noise terms in the above master equations in the leading order of $\Omega_{13}, \Omega_{23}$, we rewrite the power spectrum defined in the main text without the noise averaging:

$$
\begin{aligned}
P(\omega) & =\frac{1}{2 \pi} \int_{-\infty}^{\infty} d t e^{i \omega t} \tilde{\rho}_{21}^{\dagger}(t) \tilde{\rho}_{21}(0) \\
& =\frac{1}{2 \pi} \int_{-\infty}^{\infty} d t e^{i\left(\omega-\omega_{s_{1}}+\omega_{s_{2}}\right) t} \rho_{21}^{\dagger}(t) \rho_{21}(0) .
\end{aligned}
$$

When the driven atoms reach the steady state at a long time, $\rho_{21}(t)$ becomes time independent. Then we can replace $\rho_{21}(t)$ and $\rho_{21}(0)$ by their steady-state value $\rho_{21}$ to find

$$
\begin{aligned}
P(\omega) & =\frac{1}{2 \pi} \int_{-\infty}^{\infty} d t e^{i\left(\omega-\omega_{s_{1}}+\omega_{s_{2}}\right) t}\left|\rho_{21}\right|^{2} \\
& =\delta\left(\omega+\omega_{s_{2}}-\omega_{s_{1}}\right)\left|\rho_{21}\right|^{2} .
\end{aligned}
$$


In the main text, we provide a relatively simple formula for $\rho_{21}$ by choosing $\Omega_{13}=\Omega_{23}=\Omega$ and $\Delta_{23}=0, \Delta_{13}=\Delta$, and that formula is employed for the driven thermal vapors.
Here, we give a more general formula when $\Delta_{23} \neq 0$, which is our experimental condition for the driven rubidium cold atoms:

$$
\rho_{21}=\frac{\gamma \Omega^{2}\left(\tilde{\Delta}(2 i \gamma+\tilde{\Delta})-4 \Omega^{2}+(-2 \gamma+i \tilde{\Delta}) \gamma^{\prime}\right)}{\gamma\left(\tilde{\Delta}^{2}\left(2 \gamma^{2}+\Delta_{23}^{2}+\Delta_{13}^{2}\right)+2 \Omega^{2} \tilde{\Delta}^{2}+8 \Omega^{4}\right)+\Omega^{2}\left(8 \gamma^{2}+\left(\Delta_{23}+\Delta_{13}\right)^{2}+12 \Omega^{2}\right) \gamma^{\prime}+\gamma \gamma^{\prime 2}\left(2 \gamma^{2}+\Delta_{23}^{2}+\Delta_{13}^{2}+6 \Omega^{2}\right)},
$$

where $\tilde{\Delta}=\Delta_{23}-\Delta_{13}$. The power spectrum in Eq. (B12) gives a delta peak (broadened in our experiment by relative frequency jitter of the two Raman fields derived from two independent AOMs) at $\omega=\omega_{s_{1}}-\omega_{s_{2}}$, whose strength is determined by $\left|\rho_{21}\right|^{2}$ given in Eq. (B13). The strength of the peak is maximum at $\Delta_{13}=0$ when $\Delta_{23}=0$, and the peak height falls with increasing $\left|\Delta_{13}\right|$ as shown in Fig. 12. For $\Delta_{23}=0$, the envelope of the sharp delta peaks with changing $\Delta_{13}$ has a Lorentzian-like shape when $\Omega<\gamma$ but it changes to a Gaussian-like form for larger $\Omega$. In the inset of Fig. 12, we further show the dependence of the envelope width of the driven power spectrum as a function of the (scaled) driving Rabi frequency $\Omega / \gamma$. As intuitively expected, the envelope width falls with decreasing driving Rabi frequency, which we have also measured experimentally in thermal vapor [see Fig. 3(b)]. For $\Delta_{23} \neq 0$, the peak height is maximum around $\Delta_{13}=\Delta_{23}$.

\section{APPENDIX C: DETAILS OF THE DATA ANALYSIS PROCEDURE}

For a homogeneous magnetic field as in the thermal vapor measurements, the Faraday rotation fluctuations signal (both the intrinsic and the Raman driven) is centered around a single

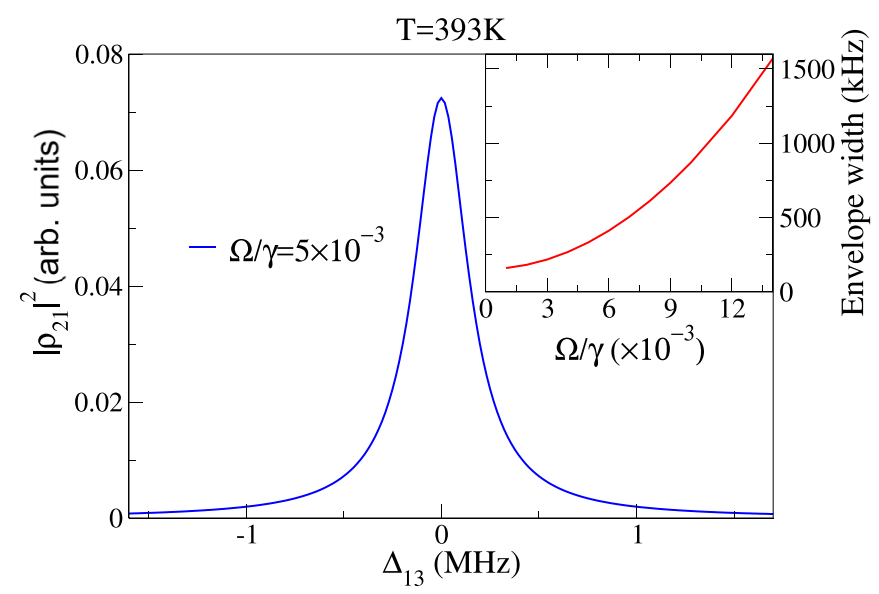

FIG. 12. The envelope $\left|\rho_{21}\right|^{2}$ of the Raman driven power spectrum (in arb. units) from rubidium atomic vapor with the Raman beam detuning $\Delta_{13}$ at relatively low driving Rabi frequency $\Omega / \gamma=$ $5 \times 10^{-3}$ and temperature. $\mathrm{T}=393 \mathrm{~K}$ where $\Delta_{23}=0$. The inset shows the dependence of the envelope width (with a factor of $2 \pi$ ) of the driven power spectrum as a function of the (scaled) driving Rabi frequency $\Omega / \gamma$.
Larmor frequency $v_{L}\left(=g_{F} \mu_{B} B / h\right.$, where $g_{F}$ is the Landé $g$ factor of the hyperfine $F$-levels, $\mu_{B}$ is the Bohr magneton, and $h$ is the Planck's constant), determined by the magnetic field strength $B$. However, for the cold atoms inside a MOT, the Larmor frequency $v_{L}(x)$ varies over space along $\hat{x}$. Therefore, the Zeeman splittings of the ground hyperfine levels are determined by the magnitude of the local magnetic field and the Raman resonance condition is also position-dependent, i.e., $\delta_{12}(x)=v_{L}(x)$. In our numerical modeling, we take this position-dependent local magnetic field into account to calculate the strength of the Faraday rotation fluctuations signal at different frequency.

Moreover, the orientation $\left[\theta_{B}(x)\right]$ of the local magnetic field also varies along $\hat{x}$ inside the MOT. The SN signal strength at $v_{L}(x)$ also gets modified by a factor $\sin ^{2} \theta_{B}(x)$ [16]. We have experimentally verified this correction factor by performing a separate calibration measurement of the intrinsic $\mathrm{SN}$ spectrum in thermal atoms.

The atom density distribution within the MOT detected by the probe laser is not uniform, which is evident from the absorption image in Fig. 10. We incorporate this density distribution in the modeling of the SN signal strength $[\propto n(x)$, where $n(x)$ is the number density of atoms at position $x$ ] from the MOT $[17,53]$. Note that $n(x)$ can be measured precisely using absorption imaging.

Another minor correction to the SN signal strength can be from the definition of the quantization axis, which also varies along $x$-direction on the $x-z$ plane. Since the local magnetic field alters over space, the coupling of the Raman fields with the atoms in the MOT also depends on the $x$ position. Such correction can be incorporated in our modeling by an $x$-dependent Rabi frequency defined as $\Omega(x)=$ $\Omega\left(1-\sin ^{2} \theta_{B}(x) / 2\right)$.

So far, we have discussed the Raman-driven spin coherence between the ground states involving a single $\Lambda$ system formed by states $|i\rangle$, where $i=1,2$, and 3 , as described in the paper. Ideally, in cold atom experiments, six $\Lambda$ systems are involved in giving rise to the driven power spectrum generated from $F=3$ and $F^{\prime}=4$ hyperfine levels of ${ }^{85} \mathrm{Rb}$. However, the value of the Landé $g$ factor is different for those two hyperfine levels, resulting in a dissimilar contribution in building the signal strength from the individual $\Lambda$ system through the optical detuning of the Raman fields.

Incorporating the above factors and corrections, we get strength $\left|\rho_{21}(x)\right|^{2}$ of the Faraday rotation fluctuation signals from the atoms at position $x$ inside the MOT:

$$
\left|\rho_{21}(x)\right|^{2}=\left|\rho_{21}(\Omega(x))\right|^{2} n(x) \sin ^{2} \theta_{B}(x) .
$$


For a fixed position $x$ within the MOT, we consider the contributions of all six $\Lambda$ systems and add them up to get the total driven spectrum strength. The six $\Lambda$ systems contribute differently via the optical detuning $\Delta_{23}(n)$ of the Raman fields from the excited state, i.e.,

$$
\Delta_{23}(n)=2 \gamma+(3-n)\left(g_{F^{\prime}}-g_{F}\right) \mu_{B} B(x) / h,
$$

where $n$ runs from 1 to 6 and $g_{F^{\prime}}$ is the Landé $g$ factor of the excited level. We fix the detuning $\Delta_{23}(n)=$
$2 \gamma$ for $n=3$ in our experiment as described in the main text.

In Figs. 4(c)-4(e) of the main text, we present some plots for the Faraday rotation fluctuations signals as a function of $\delta_{12}$ from a driven cold rubidium cloud. We have fitted the experimental data using Eq. (C1) along with $\rho_{21}(\Omega(x))$ from Eq. (B13), where $\Omega$ is replaced by $\Omega(x)$ and we employ $\Delta_{23}$ from Eq. (C2). The only free parameter in this fitting is the relaxation rate $\gamma^{\prime}$ of the hyperfine ground-level Zeeman states, and all other parameters are measured in our experiments.
[1] L. Pezzè, A. Smerzi, M. K. Oberthaler, R. Schmied, and P. Treutlein, Quantum metrology with nonclassical states of atomic ensembles, Rev. Mod. Phys. 90, 035005 (2018).

[2] K. Hammerer, A. S. Sørensen, and E. S. Polzik, Quantum interface between light and atomic ensembles, Rev. Mod. Phys. 82, 1041 (2010).

[3] D. Suter and G. A. Álvarez, Colloquium: Protecting quantum information against environmental noise, Rev. Mod. Phys. 88, 041001 (2016).

[4] C. L. Degen, F. Reinhard, and P. Cappellaro, Quantum sensing, Rev. Mod. Phys. 89, 035002 (2017).

[5] M. W. Mitchell and S. Palacios Alvarez, Colloquium: Quantum limits to the energy resolution of magnetic field sensors, Rev. Mod. Phys. 92, 021001 (2020).

[6] L. Bányai and S. W. Koch, Semiconductor Quantum Dots (World Scientific, Singapore, 1993).

[7] M. Atatüre, J. Dreiser, A. Badolato, and A. Imamoglu, Observation of Faraday rotation from a single confined spin, Nat. Phys. 3, 101 (2007).

[8] M. H. Mikkelsen, J. Berezovsky, N. G. Stoltz, L. A. Coldren, and D. D. Awschalom, Optically detected coherent spin dynamics of a single electron in a quantum dot, Nat. Phys. 3, 770 (2007).

[9] R. Yamamoto, J. Kobayashi, K. Kato, T. Kuno, Y. Sakura, and Y. Takahashi, Site-resolved imaging of single atoms with a Faraday quantum gas microscope, Phys. Rev. A 96, 033610 (2017).

[10] M. A. Kristensen, M. Gajdacz, P. L. Pedersen, C. Klempt, J. F. Sherson, J. J. Arlt, and A. J. Hilliard, Sub-atom shot noise Faraday imaging of ultracold atom clouds, J. Phys. B 50, 034004 (2017).

[11] M. Gajdacz, P. L. Pedersen, T. Mørch, A. J. Hilliard, J. Arlt, and J. F. Sherson, Non-destructive Faraday imaging of dynamically controlled ultracold atoms, Rev. Sci. Instrum. 84, 083105 (2013).

[12] D. Yang, C. Laflamme, D. V. Vasilyev, M. A. Baranov, and P. Zoller, Theory of a Quantum Scanning Microscope for Cold Atoms, Phys. Rev. Lett. 120, 133601 (2018).

[13] J. Meineke, J.-P. Brantut, D. Stadler, T. Müller, H. Moritz, and T. Esslinger, Interferometric measurement of local spin fluctuations in a quantum gas, Nat. Phys. 8, 454 (2012).

[14] V. S. Zapasskii, Spin-noise spectroscopy: from proof of principle to applications, Adv. Opt. Photon. 5, 131 (2013).

[15] G. G. Kozlov, A. A. Fomin, M. Y. Petrov, I. I. Ryzhov, and V. S. Zapasskii, Raman scattering model of the spin noise, Opt. Express 29, 4770 (2021).
[16] N. A. Sinitsyn and Y. V. Pershin, The theory of spin noise spectroscopy: a review, Rep. Prog. Phys. 79, 106501 (2016).

[17] S. A. Crooker, D. G. Rickel, A. V. Balatsky, and D. L. Smith, Spectroscopy of spontaneous spin noise as a probe of spin dynamics and magnetic resonance, Nature (London) 431, 49 (2004).

[18] M. Swar, D. Roy, D. D, S. Chaudhuri, S. Roy, and H. Ramachandran, Measurements of spin properties of atomic systems in and out of equilibrium via noise spectroscopy, Opt. Express 26, 32168 (2018).

[19] V. G. Lucivero, R. Jiménez-Martínez, J. Kong, and M. W. Mitchell, Squeezed-light spin noise spectroscopy, Phys. Rev. A 93, 053802 (2016).

[20] Y. Tang, Y. Wen, L. Cai, and K. Zhao, Spin-noise spectrum of hot vapor atoms in an anti-relaxation-coated cell, Phys. Rev. A 101, 013821 (2020).

[21] M. Oestreich, M. Römer, R. J. Haug, and D. Hägele, Spin Noise Spectroscopy in GaAs, Phys. Rev. Lett. 95, 216603 (2005).

[22] D. Roy, Y. Li, A. Greilich, Y. V. Pershin, A. Saxena, and N. A. Sinitsyn, Spin noise spectroscopy of quantum dot molecules, Phys. Rev. B 88, 045320 (2013).

[23] M. M. Glazov, Coherent spin dynamics in semiconductor quantum dots, J. Appl. Phys. 113, 136503 (2013).

[24] D. Roy, L. Yang, S. A. Crooker, and N. A. Sinitsyn, Cross-correlation spin noise spectroscopy of heterogeneous interacting spin systems, Sci. Rep. 5, 9573 (2015).

[25] O. Katz, O. Peleg, and O. Firstenberg, Coherent Coupling of Alkali Atoms by Random Collisions, Phys. Rev. Lett. 115, 113003 (2015).

[26] M. M. Glazov, M. A. Semina, E. Y. Sherman, and A. V. Kavokin, Spin noise of exciton polaritons in microcavities, Phys. Rev. B 88, 041309(R) (2013).

[27] I. I. Ryzhov, V. O. Kozlov, N. S. Kuznetsov, I. Y. Chestnov, A. V. Kavokin, A. Tzimis, Z. Hatzopoulos, P. G. Savvidis, G. G. Kozlov, and V. S. Zapasskii, Spin noise signatures of the selfinduced Larmor precession, Phys. Rev. Research 2, 022064(R) (2020).

[28] I. I. Ryzhov, G. G. Kozlov, D. S. Smirnov, M. M. Glazov, Y. P. Efimov, S. A. Eliseev, V. A. Lovtcius, V. V. Petrov, K. V. Kavokin, A. V. Kavokin, and V. S. Zapasskii, Spin noise explores local magnetic fields in a semiconductor, Sci. Rep. 6, 21062 (2016).

[29] A. Recati and S. Stringari, Spin Fluctuations, Susceptibility, and the Dipole Oscillation of a Nearly Ferromagnetic Fermi Gas, Phys. Rev. Lett. 106, 080402 (2011). 
[30] E. Altman, E. Demler, and M. D. Lukin, Probing many-body states of ultracold atoms via noise correlations, Phys. Rev. A 70, 013603 (2004).

[31] D. Roy, R. Singh, and R. Moessner, Probing many-body localization by spin noise spectroscopy, Phys. Rev. B 92, 180205(R) (2015).

[32] P. N. Jepsen, J. Amato-Grill, I. Dimitrova, W. W. Ho, E. Demler, and W. Ketterle, Spin transport in a tunable Heisenberg model realized with ultracold atoms, Nature (London) 588, 403 (2020).

[33] Y. Cohen, K. Jadeja, S. Sula, M. Venturelli, C. Deans, L. Marmugi, and F. Renzoni, A cold atom radiofrequency magnetometer, Appl. Phys. Lett. 114, 073505 (2019).

[34] K. Gosar, T. Arh, T. Mežnaršič, I. Kvasič, D. Ponikvar, T. Apih, R. Kaltenbaek, R. Žitko, E. Zupanič, S. Beguš, and P. Jeglič, Single-shot Stern-Gerlach magnetic gradiometer with an expanding cloud of cold cesium atoms, Phys. Rev. A 103, 022611 (2021).

[35] I. Bloch, J. Dalibard, and W. Zwerger, Many-body physics with ultracold gases, Rev. Mod. Phys. 80, 885 (2008).

[36] M. Scully and M. Zubairy, Quantum Optics (Cambridge University Press, United Kingdom, 1997).

[37] D. A. Steck, Rubidium 87 D Line Data, http://steck.us/ alkalidata/rubidium87numbers.pdf.

[38] D. A. Steck, Rubidium 85 D Line Data, http://steck.us/ alkalidata.

[39] For our measurements, any relative phase difference between $\Omega_{13}$ and $\Omega_{23}$ is irrelevant.

[40] Note that $2 \pi \delta_{12}=2 \pi v_{L}-\left(\Delta_{23}-\Delta_{13}\right)$.

[41] The pressure broadened line width, $\gamma=2 \pi \times 1.79(0.02) \mathrm{GHz}$, measured by absorption spectroscopy.

[42] A. A. Fomin, M. Y. Petrov, G. G. Kozlov, M. M. Glazov, I. I. Ryzhov, M. V. Balabas, and V. S. Zapasskii, Spin-alignment noise in atomic vapor, Phys. Rev. Research 2, 012008(R) (2020).
[43] V. Mugundhan, M. Swar, S. Bhar, and S. Chaudhuri, A realtime digital receiver for correlation measurements in atomic systems, IEEE Trans. Instrum. Meas. 70, 1 (2021).

[44] Experimentally, we observed a small but finite width $(\sim 2 \mathrm{kHz})$ of the individual spectrum instead of the delta function as predicted in Eq. (4). This width is due to the relative frequency jitter of the two Raman fields derived from two independent AOMs.

[45] N. Behbood, F. Martin Ciurana, G. Colangelo, M. Napolitano, G. Tóth, R. J. Sewell, and M. W. Mitchell, Generation of Macroscopic Singlet States in a Cold Atomic Ensemble, Phys. Rev. Lett. 113, 093601 (2014).

[46] R. Thomas, J. S. Otto, M. Chilcott, A. B. Deb, and N. Kjærgaard, Reducing number fluctuations in an ultracold atomic sample using Faraday rotation and iterative feedback, arXiv:2102.01773.

[47] Y. Liu, E. Gomez, S. E. Maxwell, L. D. Turner, E. Tiesinga, and P. D. Lett, Number Fluctuations and Energy Dissipation in Sodium Spinor Condensates, Phys. Rev. Lett. 102, 225301 (2009).

[48] M. Jasperse, Faraday magnetic resonance imaging of BoseEinstein condensates, Ph. D. thesis, Monash University, Australia, 2017.

[49] B. Tekin, SternGerlach experiment with higher spins, Eur. J. Phys. 37, 035401 (2016).

[50] O. Katz and O. Firstenberg, Transverse optical pumping of spin states, Commun. Phys. 2, 58 (2019).

[51] A. Ben-Kish and M. V. Romalis, Dead-Zone-Free Atomic Magnetometry with Simultaneous Excitation of Orientation and Alignment Resonances, Phys. Rev. Lett. 105, 193601 (2010).

[52] C. I. James Simpson, John Lane, and R. Youngquist, Technical Report Nos. 1-3 (2001).

[53] M. Swar, D. Roy, D. Dhanalakshmi, S. Chaudhuri, S. Roy, and H. Ramachandran, Measurements of Temporal Fluctuations of Magnetization in Alkali Vapor and Applications, Emerging Trends in Advanced Spectroscopy (River Publishers Series in Optics \& Photonics, Denmark, 2019). 